\title{
A Novel High-Throughput Chaotic Advection Microreactor for the Preparation of Uniform BaSO4 Nanoparticles
}

\author{
Hua Yang ${ }^{1}$, Shi-Xiao Wei ${ }^{1}$, Han Chen ${ }^{1}$, Lang Chen $^{2}$, Chak-tong Au ${ }^{3}$, Ting-Liang Xie ${ }^{1}$, and \\ Shuang-Feng Yin ${ }^{1}$ \\ ${ }^{1}$ Affiliation not available \\ ${ }^{2}$ Hunan University \\ ${ }^{3}$ National Engineering Research Center of Chemical Fertilizer Catalyst
}

December 27, 2021

\begin{abstract}
Owing to high mixing efficiency, microreactors are used to synthesize uniform BaSO4 nanoparticles, but application in industrial scale is limited due to poor throughput. In this work, a high-throughput passive four-stage asymmetric oscillating feedback microreactor using chaotic mixing mechanism was developed to prepare BaSO4 nanoparticles of high size uniformity. Threedimensional unsteady simulations showed that chaotic mixing could be induced by three unique secondary flows (i.e., vortex, recirculation, and oscillation), and the fluid oscillation mechanism was examined in detail. Simulations and Villermaux-Dushman experiments indicate that almost complete mixing in molecular level could be achieved when total volumetric flow rate Qtotal was larger than $10 \mathrm{~mL} / \mathrm{min}$, and the prepared BaSO4 nanoparticles were with narrow particle size distribution (PSD). Through the adjustment of Qtotal and reactant concentrations, it is easy to control the average size. An average size of $26 \mathrm{~nm}$ with narrow PSD could be achieved at Qtotal $=160 \mathrm{~mL} / \mathrm{min}$.
\end{abstract}

\section{Hosted file}

Manuscript.docx available at https://authorea.com/users/452747/articles/550705-a-novelhigh-throughput-chaotic-advection-microreactor-for-the-preparation-of-uniform-baso4nanoparticles 

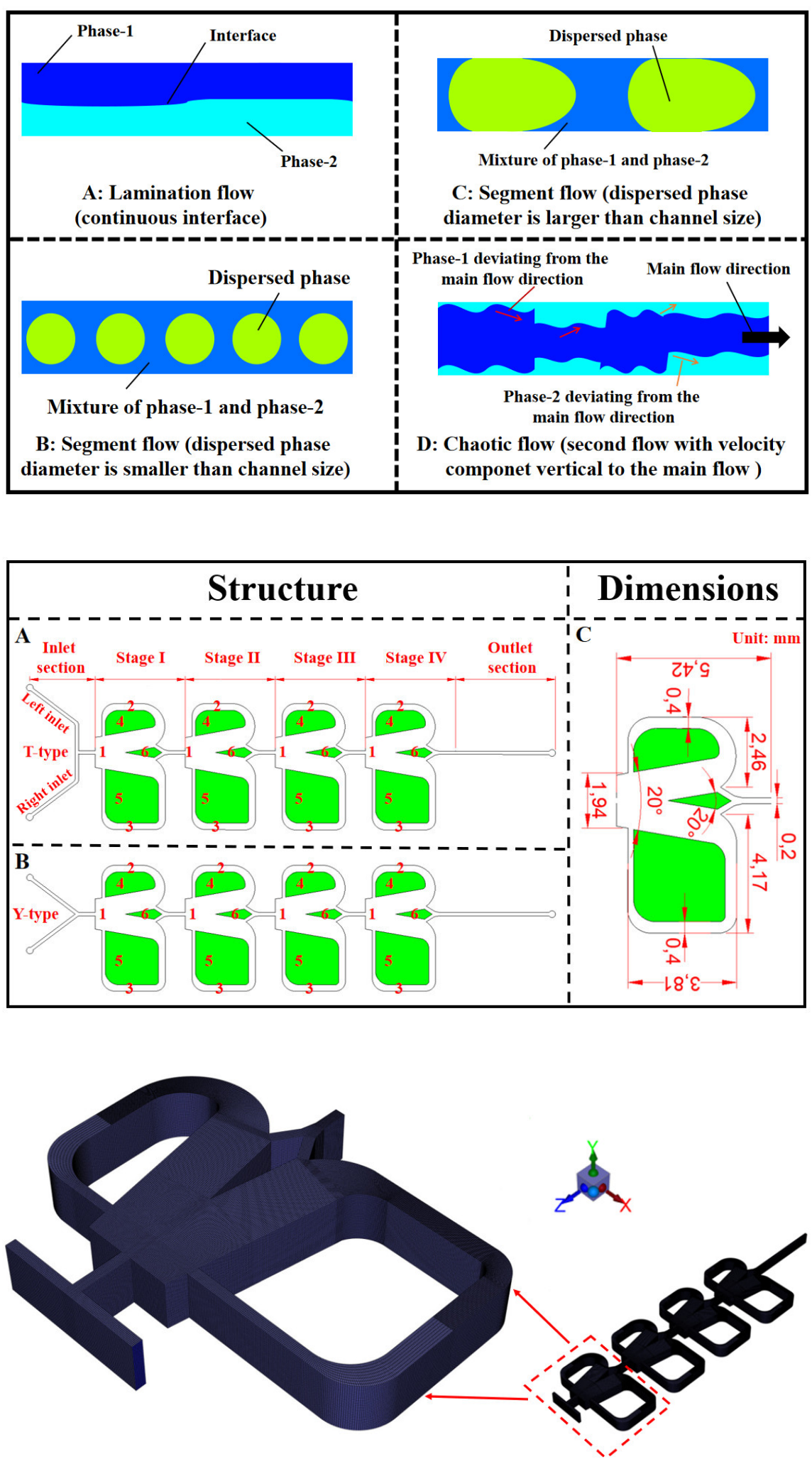

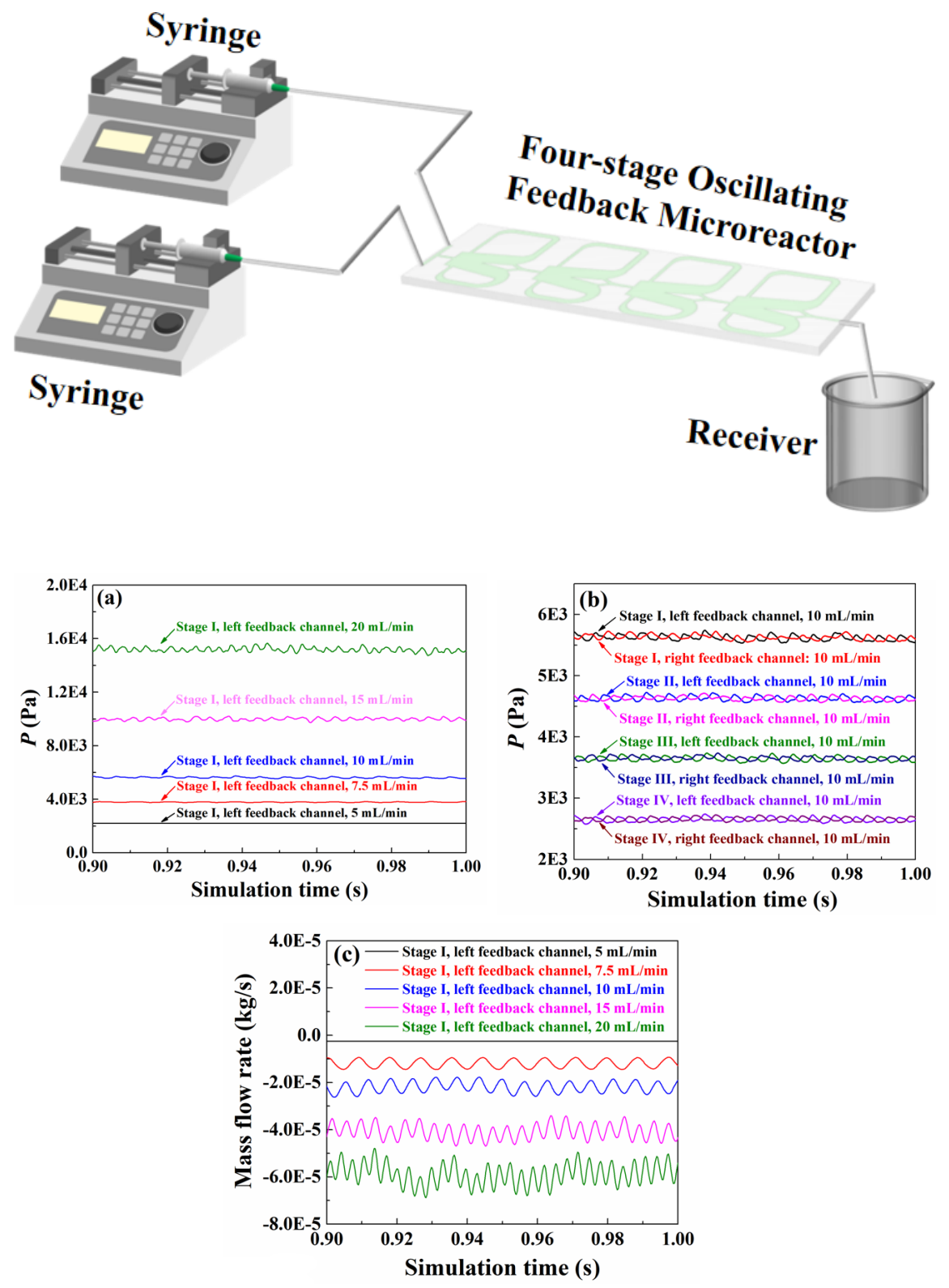

Re
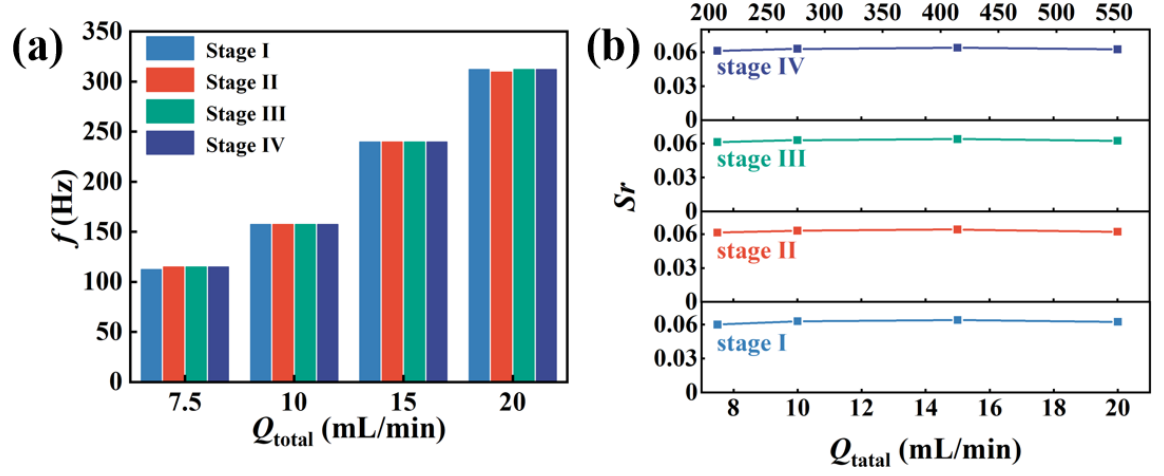

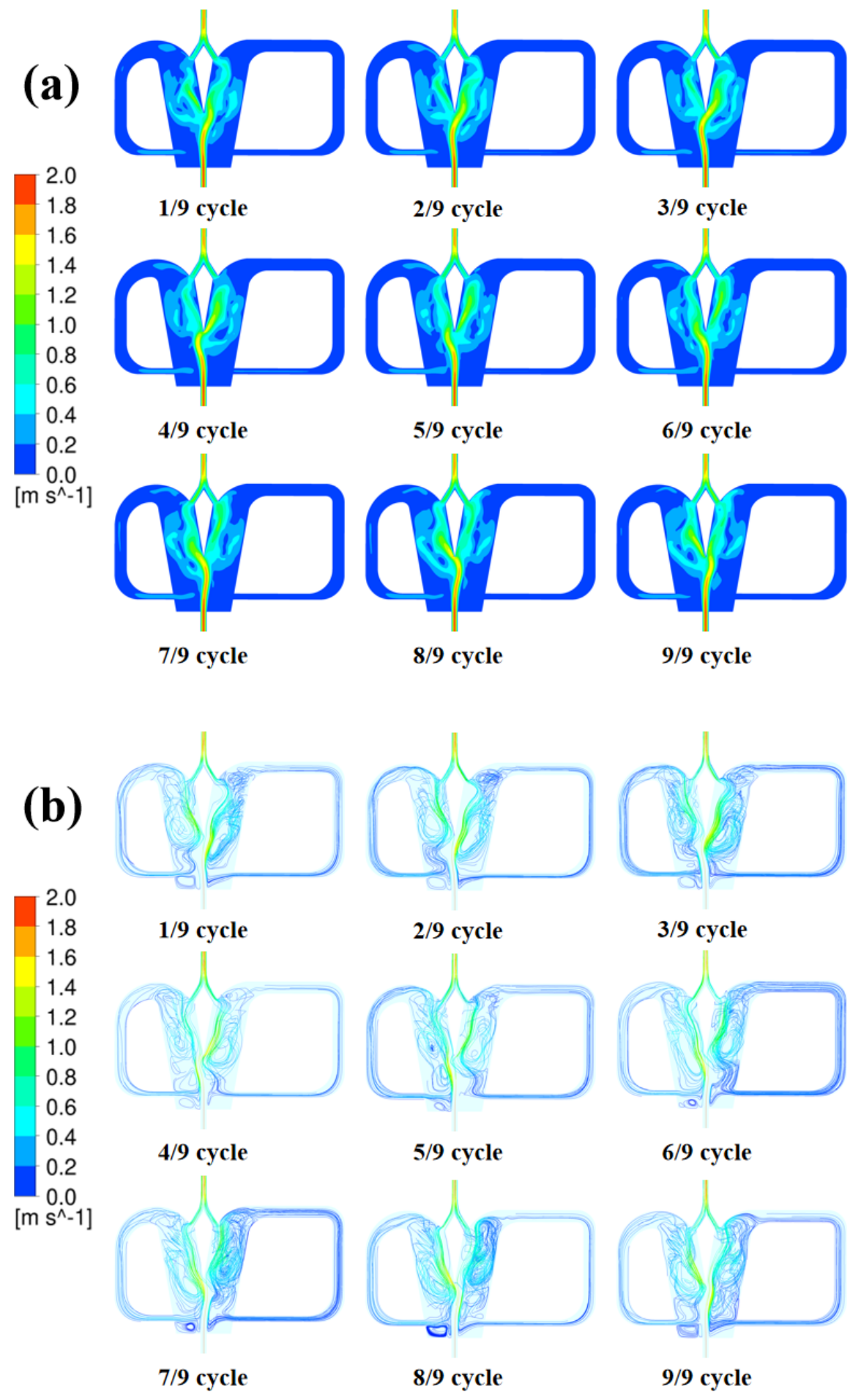


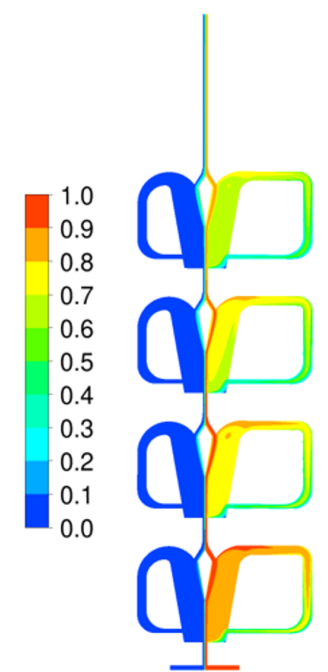

(a)

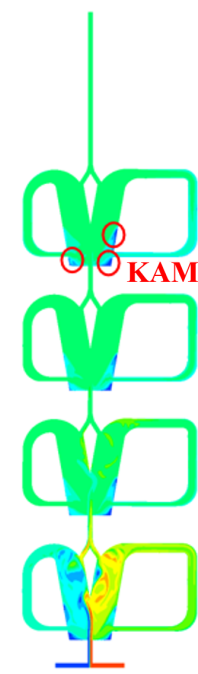

(b)

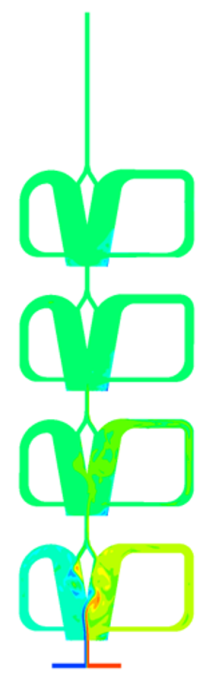

(c)

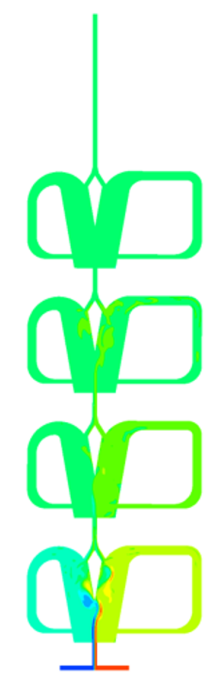

(d)

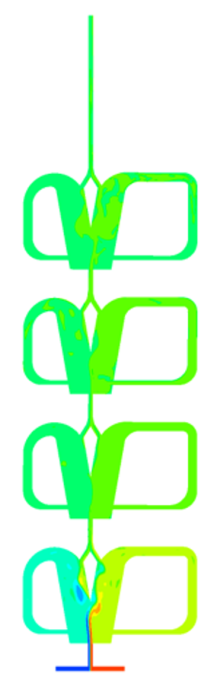

(e)

(a)

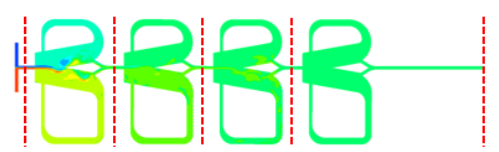

(d) $\begin{array}{llll}\mathbf{S}_{0} & \mathrm{~S}_{1} & \mathrm{~S}_{2} & \mathrm{~S}_{3}\end{array}$

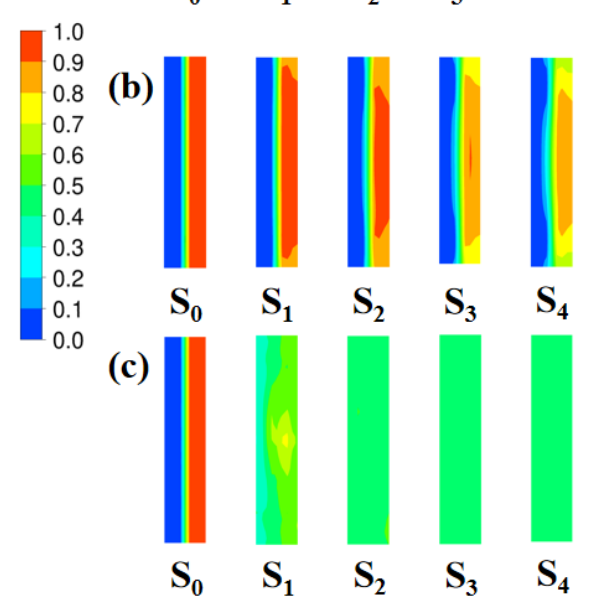

$\mathbf{S}_{4}$

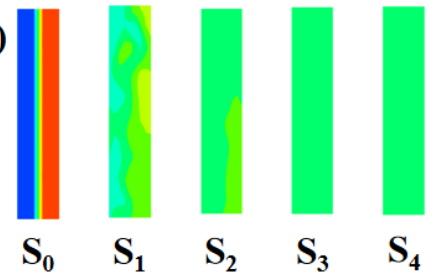

(e)

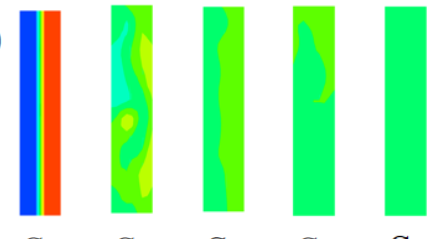

(f)

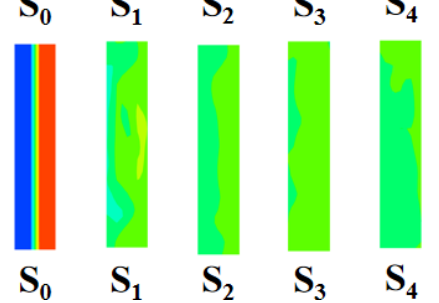



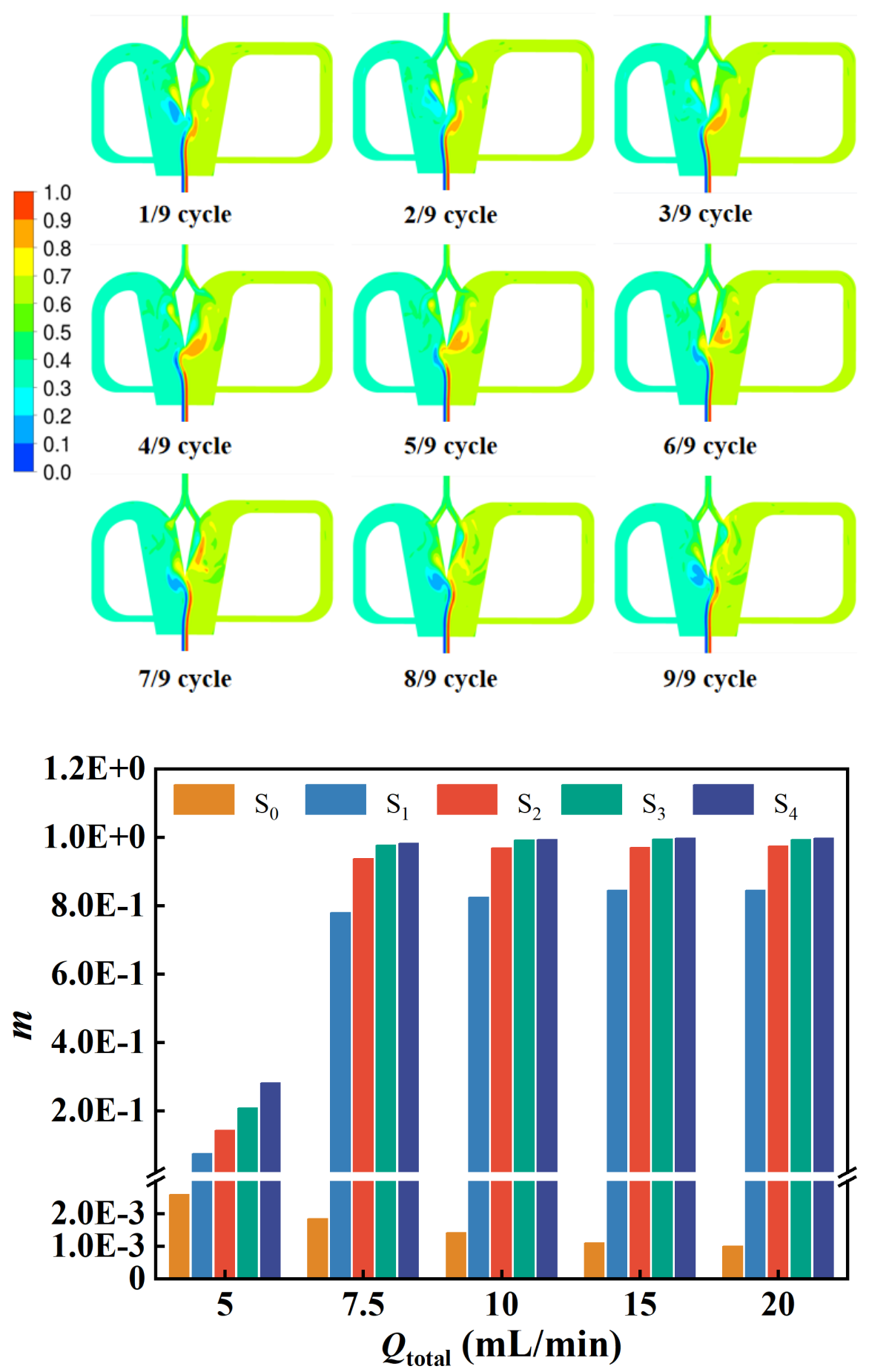

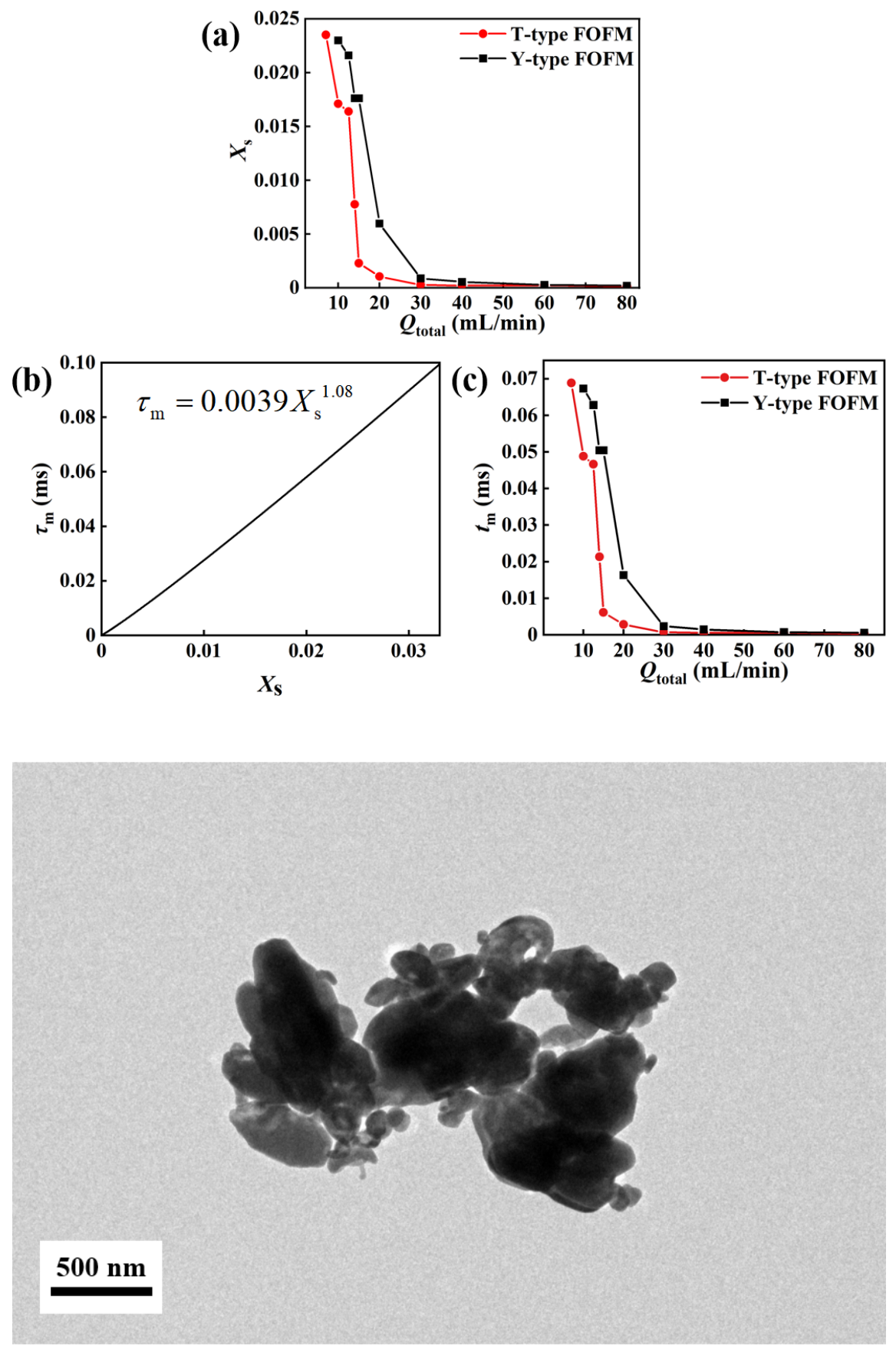


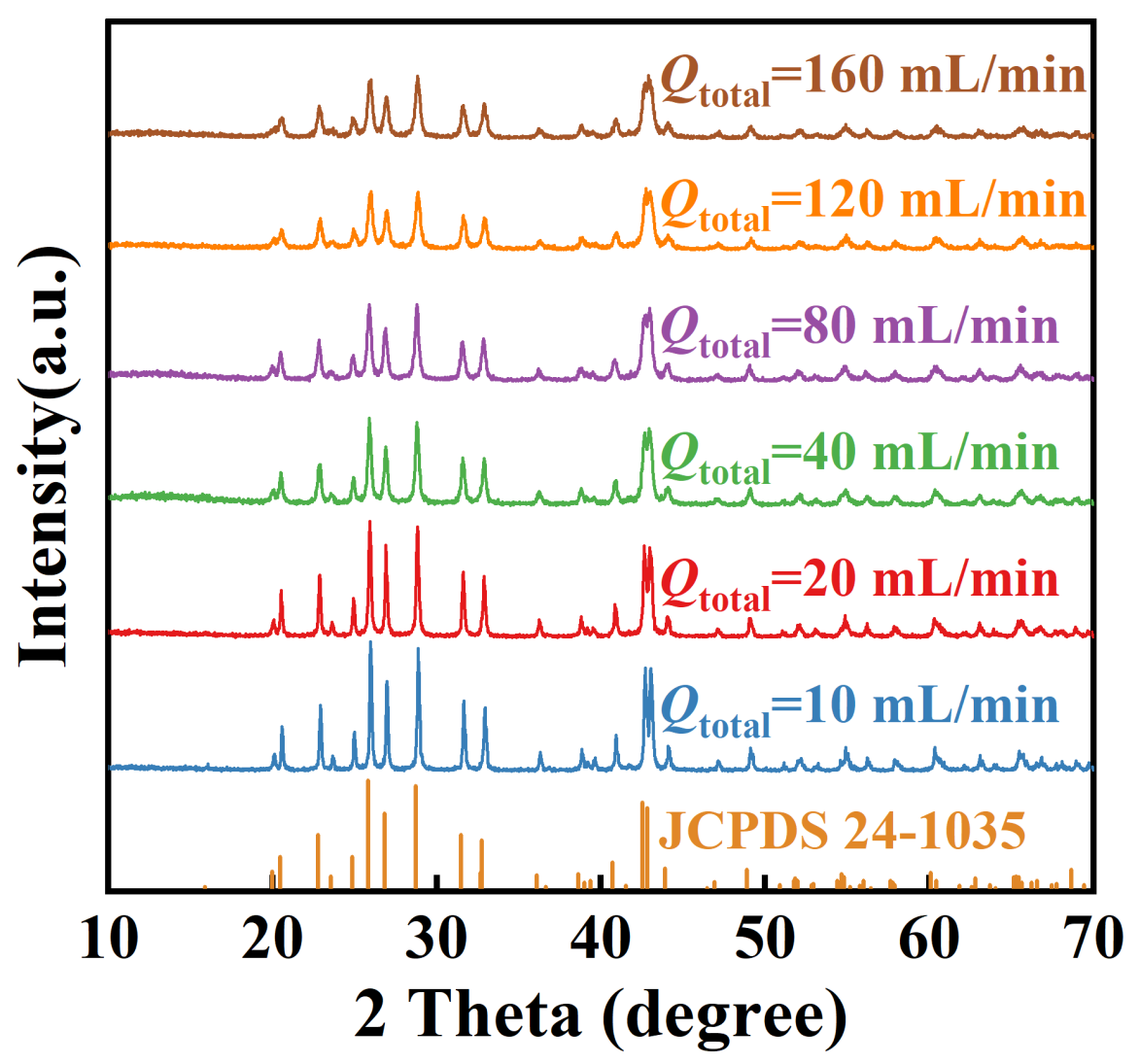



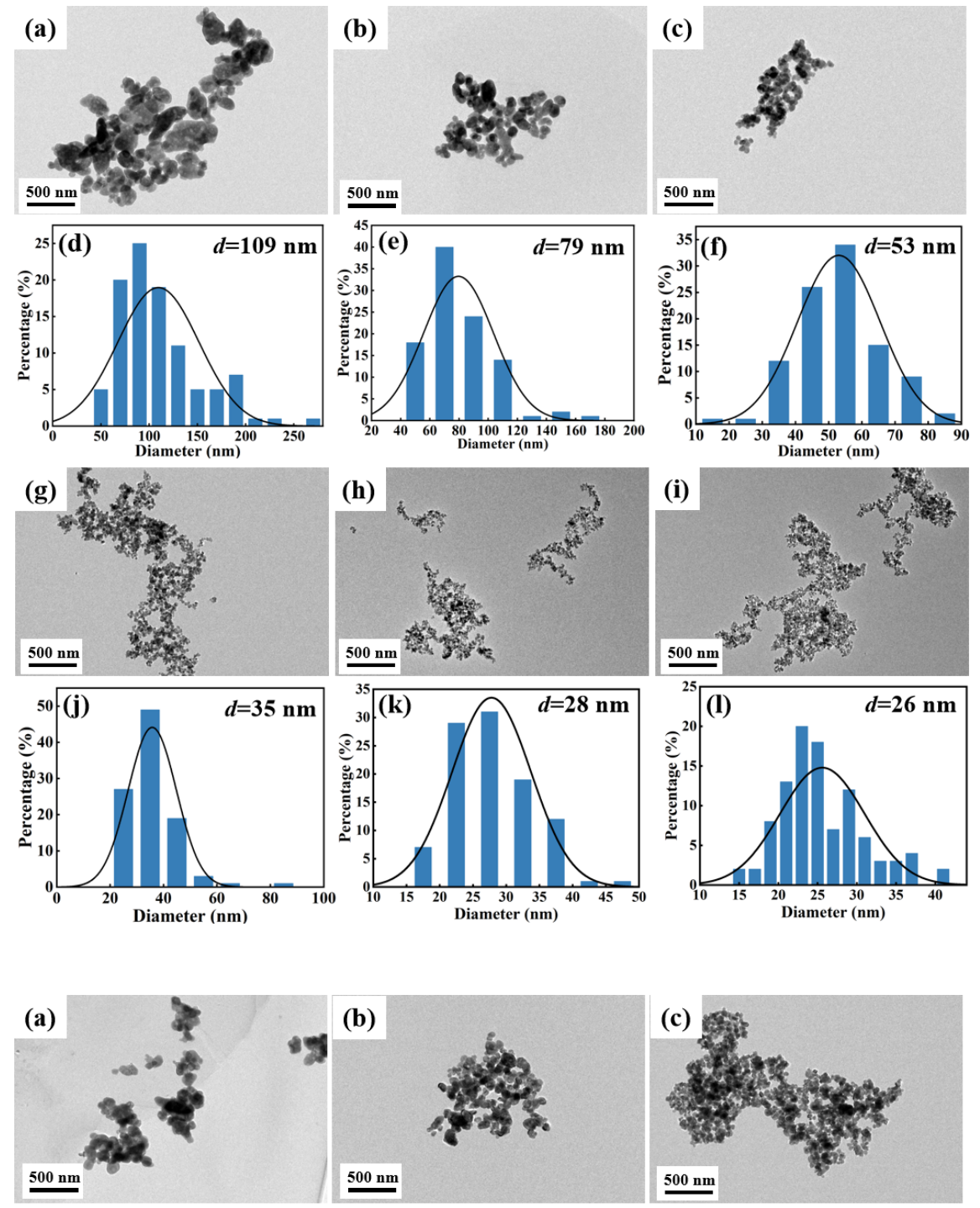

(b)
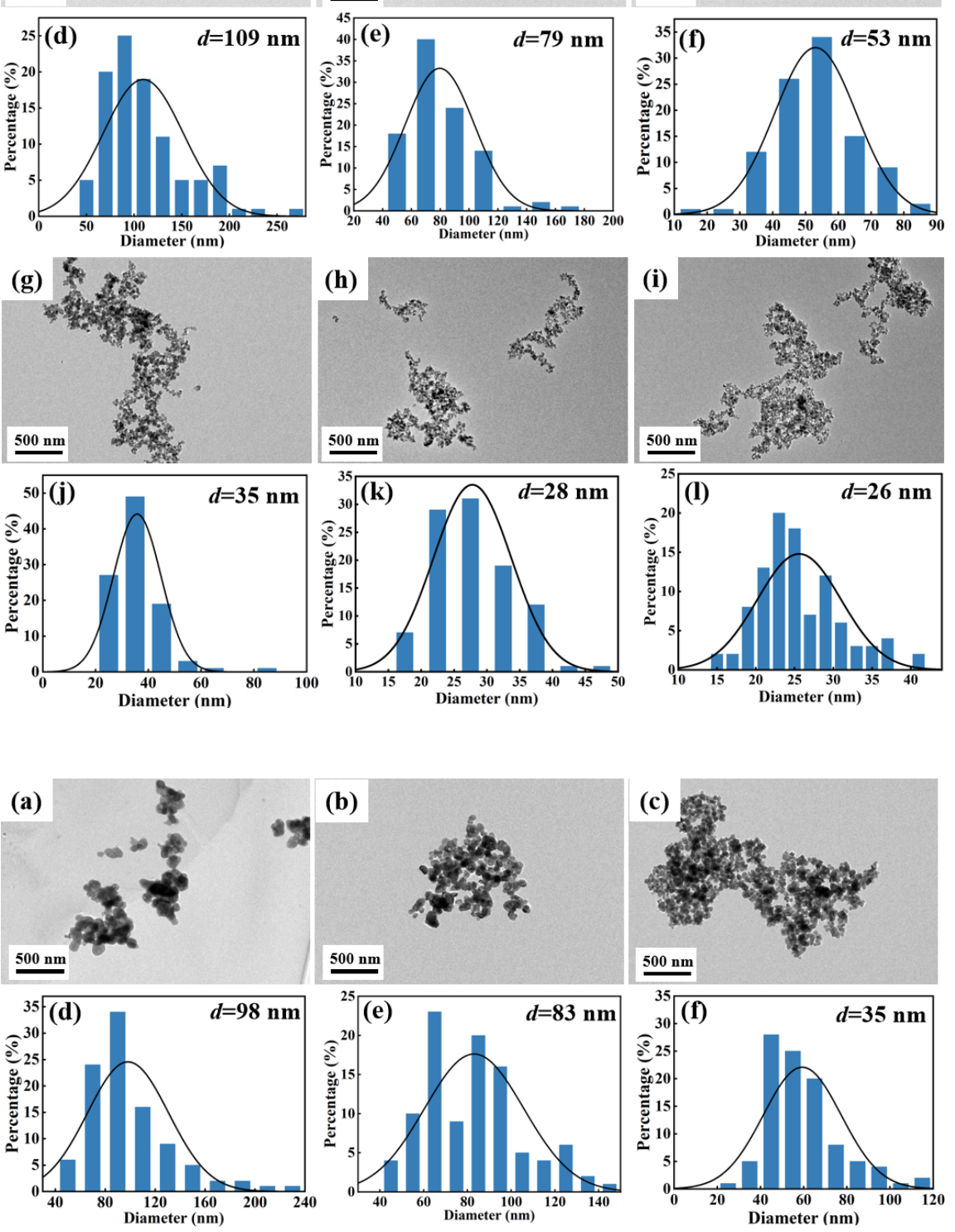

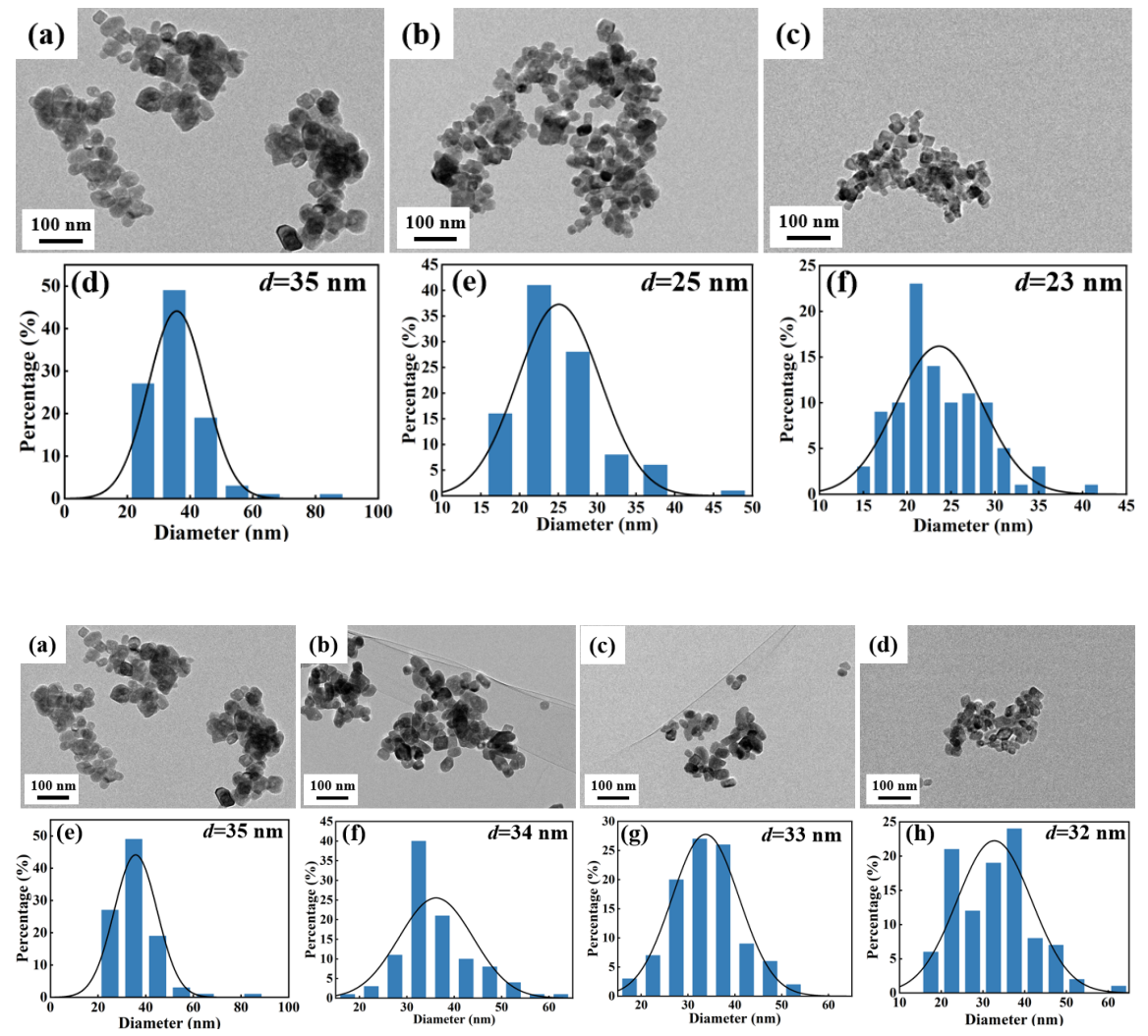

\section{Hosted file}

Table.docx available at https://authorea.com/users/452747/articles/550705-a-novelhigh-throughput-chaotic-advection-microreactor-for-the-preparation-of-uniform-baso4nanoparticles 